ACCEPTED MANUSCRIPT

\title{
Nanoscale axial position and orientation measurement of hexagonal boron nitride quantum emitters using a tunable nanophotonic environment
}

To cite this article before publication: Pankaj K Jha et al 2021 Nanotechnology in press https://doi.org/10.1088/1361-6528/ac2b71

\section{Manuscript version: Accepted Manuscript}

Accepted Manuscript is "the version of the article accepted for publication including all changes made as a result of the peer review process, and which may also include the addition to the article by IOP Publishing of a header, an article ID, a cover sheet and/or an 'Accepted

Manuscript' watermark, but excluding any other editing, typesetting or other changes made by IOP Publishing and/or its licensors"

This Accepted Manuscript is (C) 2021 IOP Publishing Ltd.

During the embargo period (the 12 month period from the publication of the Version of Record of this article), the Accepted Manuscript is fully protected by copyright and cannot be reused or reposted elsewhere.

As the Version of Record of this article is going to be / has been published on a subscription basis, this Accepted Manuscript is available for reuse under a CC BY-NC-ND 3.0 licence after the 12 month embargo period.

After the embargo period, everyone is permitted to use copy and redistribute this article for non-commercial purposes only, provided that they adhere to all the terms of the licence https://creativecommons.org/licences/by-nc-nd/3.0

Although reasonable endeavours have been taken to obtain all necessary permissions from third parties to include their copyrighted content within this article, their full citation and copyright line may not be present in this Accepted Manuscript version. Before using any content from this article, please refer to the Version of Record on IOPscience once published for full citation and copyright details, as permissions will likely be required. All third party content is fully copyright protected, unless specifically stated otherwise in the figure caption in the Version of Record.

View the article online for updates and enhancements. 


\title{
Nanoscale axial position and orientation measurement of hexagonal boron nitride quantum emitters using a tunable nanophotonic environment
}

\author{
Pankaj K. Jha ${ }^{1, \dagger}$, Hamidreza Akbari ${ }^{1, \dagger}$, Yonghwi Kim ${ }^{1, \dagger}$, Souvik Biswas ${ }^{1}$, Harry A. Atwater ${ }^{1, *}$ \\ ${ }^{1}$ Thomas J. Watson Laboratory of Applied Physics and Materials Science, California Institute of \\ Technology, Pasadena, CA 91125, USA. \\ ${ }^{2}$ Resnick Sustainability Institute, California Institute of Technology, Pasadena, CA 91125, USA. \\ ${ }^{3}$ Joint Center for Artificial Photosynthesis, California Institute of Technology, Pasadena, CA \\ 91125, USA. \\ These authors contributed equally to this work. \\ *Corresponding author: Harry A. Atwater (haa@caltech.edu)
}

(Dated: September 19, 2021)

\section{Abstract}

Color centers in hexagonal boron nitride $(h \mathrm{BN})$ have emerged as promising candidates for singlephoton emitters (SPEs) due to their bright emission characteristics at room temperature. In contrast to mono- and few-layered $h \mathrm{BN}$, color centers in multi-layered flakes show superior emission characteristics such as higher saturation counts and spectral stability. Here, we report a method for determining both the axial position and three-dimensional dipole orientation of SPEs in thick $h \mathrm{BN}$ flakes by tuning the photonic local density of states using vanadium dioxide $\left(\mathrm{VO}_{2}\right)$, a phase change material. Quantum emitters under study exhibit a strong surface-normal dipole orientation, providing some insight on the atomic structure of $h \mathrm{BN}$ SPEs, deeply embedded in thick crystals. Next, we optimized a hot pickup technique to reproducibly transfer the $h \mathrm{BN}$ flake from $\mathrm{VO}_{2} /$ Sapphire substrate onto $\mathrm{SiO}_{2} / \mathrm{Si}$ substrate and relocated the same emitters. Our approach serves as a practical method to systematically characterize SPEs in $h \mathrm{BN}$ prior to integration in quantum photonics systems. 


\section{Introduction}

Over the past few decades, point defects (1) that introduce electronic states with optical transitions, also known as color centers, have garnered great interest for quantum photonics applications, such as quantum computation and quantum information (2,3), quantum cryptography (4), and quantum sensing (5). Wide-bandgap materials, such as diamond (6), silicon carbide (7), gallium nitride (8), and zinc oxide (9) offer promising platforms for hosting quantum emitters with emission in the visible to near-infrared spectrum. However, these materials suffer from one or more intrinsic challenges such as a requirement for cryogenic temperatures, decoherence of emitted photons, optical coupling losses, and challenges associated with chip-based photonic integration. These problems have driven researchers to seek new candidate materials with fewer disadvantages $(10,11)$.

Recent discoveries of quantum light emission from two-dimensional van der Waals (vdW) layered materials (12-17) have introduced promising candidates for single photon emitters (SPEs). In contrast to bulk materials, vdW materials offer easier integration with photonic structures and minimal loss due to refractive index mismatch $(18,19)$. Among several candidate vdW host materials, hexagonal boron nitride $(h \mathrm{BN})$ has received particular attention due to its ability to offer a bright source of quantum light at room temperature. Remarkably, quantum emitters in $h \mathrm{BN}$ have shown high (> 80\%) Debye-Waller factor (17), a brightness comparable to the brightest SPEs (10, 19), polarized emission $(17,20)$, giant stark shift (21-23), magnetic-field dependent quantum emission $(24,25)$, correlated cathodoluminescence and photoluminescence emission (26), and near transform-limited optical linewidth (27), all reported at room temperature. To this date, the atomic structure of $h \mathrm{BN}$ quantum emitters is not clear; the most common approach to deduce the atomic structure of these emitters has been to compare the energy of the zero-phonon line (ZPL) and phonon-assisted emission to the first-principles calculations (28). Furthermore, to understand and quantify the intricate details of the emitter atomic structure, in particular spin-manifold, optically detected magnetic resonance (ODMR) technique has been recently reported (25). Accurate information of 3D orientation of emitting dipole would provide invaluable insight into the underlying symmetry properties of the defect center, which can complement the aforementioned approaches and help in identifying the atomic origin of emitters.

Quantum emitters in multi-layered flakes, in contrast to mono- and few-layered flakes, show superior emission characteristics such as higher saturation counts and spectral stability due 
to reduced environmental screening effects (29). Efficient coupling of these quantum emitters with nanophotonic structures would require precise information about their axial position and 3D dipole orientation. Determining, both, the 3D dipole orientation and axial position of a quantum emitter in any multi-layered $h \mathrm{BN}$ poses as a coupled problem because the polarization characteristics of detected photons is strongly influenced by either dipole orientation or axial position.

In this Letter, we demonstrate nanometer-scale axial location of $h \mathrm{BN}$ quantum emitters in a multi-layered flake by leveraging highly sensitive, distance-dependent modulation of the spontaneous emission lifetime of these quantum emitters when in close proximity to a tunable phase-change material, vanadium dioxide $\left(\mathrm{VO}_{2}\right)$. Specifically, we modify the local density of optical states (LDOS) by inducing an insulator-to-metal transition in $\mathrm{VO}_{2}$ which in turn modulates the emission rate of quantum emitters near the $h \mathrm{BN} / \mathrm{VO}_{2}$ interface. This method, taken together with emission polarimetry to determine the three-dimensional (3D) orientation of the quantum emitter, gives comprehensive information about emitters axial position and orientation, which could be used to distinguish possible candidates based on atomic structure of the emitter. Furthermore, we have optimized a polymer-assisted hot pickup and transfer process to reliably transfer $h \mathrm{BN}$ flakes from $\mathrm{VO}_{2}$ substrate onto other device substrates (here, we use $\mathrm{SiO}_{2} / \mathrm{Si}$ substrate as target), following which, the emitters under investigation were relocated. Measurement of $h \mathrm{BN}$ emitter location and 3D orientation with nanometer-scale resolution in multi-layered flakes together with advances in precise transfer, and stacking of 2D materials (30) and metal contacts (31) offer opportunities for both fundamental physics advances (32) and quantum photonic technologies $(33,34)$.

\section{Results and discussions}

The experimental configuration is shown in Figure 1(a). A quantum emitter is located at a distance $d$, within the thickness of an $h \mathrm{BN}$ flake, from the surface of a substrate that consists of a thin layer of $\mathrm{VO}_{2}$ on sapphire. Photoluminescence excitation and detection were performed with optical pumping of quantum emitters by laser excitation from the top. The excitation wavelength was set to $532 \mathrm{~nm}$. The excited quantum emitter emission decay rate depends on its interaction with the optical environment (35). By optical environment we mean the substrate beneath and the air above the $h \mathrm{BN}$ flake. We model this interaction by treating the quantum emitter as an oscillating point dipole source oriented along the direction $(\theta, \varphi)$. For an emitter in an unbounded, 
homogeneous, and lossless medium with a refractive index $n$, the spontaneous decay rate is enhanced by a factor $n$ compared to the free space. This result also holds true for bounded geometry as long as the emitter is at a distance $d \gg \lambda$ from any interface. When $d \ll \lambda$ the decay rate strongly depends on $d$, the dipole orientation $(\theta, \varphi)$, and the refractive index contrast across the interface (36-39). In this work, we manipulated the optical environment of a quantum emitter located in the vicinity of the $h \mathrm{BN} / \mathrm{VO}_{2}$ interface using $\mathrm{VO}_{2}$ whose complex refractive index exhibits a sharp change when $\mathrm{VO}_{2}$ is thermally switched from the insulating to metallic state, which occurs at near room temperature $\mathrm{T}_{\mathrm{c}} \sim 340 \mathrm{~K}(40)$.

Figure 1(b) shows the calculated relative decay rate $\beta=\gamma_{\text {Insulating }} / \gamma_{\text {Metallic }}$ of an emitter as a function of distance $d$ when the emitter is oriented perpendicular $\left(\theta=0^{\circ}\right)$ and parallel $(\theta=$ $90^{\circ}$ ) to the $h \mathrm{BN} / \mathrm{VO}_{2}$ interface. Here, $\gamma_{\text {Insulating }}$ and $\gamma_{\text {Metallic }}$ is the total (radiative and nonradiative) decay rate of the emitter when $\mathrm{VO}_{2}$ is in insulating $\left(30^{\circ} \mathrm{C}\right)$ and metallic $\left(100{ }^{\circ} \mathrm{C}\right)$ state respectively. In these simulations, we considered a flake thickness of $310 \mathrm{~nm}$ (as shown in Fig. 2(c)) and an emission wavelength of $600 \mathrm{~nm}$, corresponding to one of the quantum emitters in our experiment, shown in Figs. 2(d) and 2(e). The refractive indices of the upper medium, $h \mathrm{BN}$, and that of sapphire were set to $1,1.82(41)$, and 1.77 respectively. The complex refractive index of $\mathrm{VO}_{2}$ at $600 \mathrm{~nm}$ was extracted from our ellipsometric data and was set to $3.05+0.42 \mathrm{i}$ and $2.57+$ $0.64 \mathrm{i}$ for $\mathrm{VO}_{2}$ in insulating and metallic state respectively (Supplementary Section S1). The thickness of the $\mathrm{VO}_{2}$ layer on sapphire was set to $40 \mathrm{~nm}$ (Supplementary Section S1). In general, the photoluminescence quantum yield (PLQY) of $h \mathrm{BN}$ quantum emitters varies in the range 0.6$1.0(10,19)$ and a recent experiment has shown average PLQY in the range 0.6-0.8 for quantum emitters with zero-phonon line (ZPL) around $600 \mathrm{~nm}$ (42). The shaded area in Figure 1(b), corresponds to this PLQY range. The dashed line within the shaded regions corresponds to a PLQY $=0.79$ estimated from our experimental data (Supplementary Section S4). As can be seen in our simulation results, the relative modulation of decay rates for both orientations is clearly evident within first $\sim 50 \mathrm{~nm}$ that quickly fades away at distances $\sim 100 \mathrm{~nm}$ and above. We use this highly sensitive, distance-dependent decay rate of quantum emitters in the vicinity of the $h \mathrm{BN} / \mathrm{VO}_{2}$ interface to localize their position along the axial direction.

Figure 2(a) shows the optical microscope image of a thin $h \mathrm{BN}$ flake on a $\mathrm{VO}_{2} / \mathrm{Sapphire}$ substrate. This sample was prepared by mechanical exfoliation of high purity $h \mathrm{BN}$ single crystals 
and transferred onto a $40 \mathrm{~nm}$ thick $\mathrm{VO}_{2}$ film deposited on $500 \mu \mathrm{m}$ thick sapphire by pulsed laser deposition. To determine the thickness of this flake at each position, we employed atomic force microscopy (AFM). Figure 2(b) shows an AFM image of the $h \mathrm{BN}$ flake shown in Figure 2(a). The red dot in Figs. 2(a) and 2(b) indicates the location of the quantum emitters ' $A$ ' and ' $B$ ' with emission wavelength of $600 \mathrm{~nm}$ and $620 \mathrm{~nm}$, respectively. Figure 2(c) show AFM height profile across the lines (S-E) indicated in Fig. 2(b) where flake thickness varies in the range 230-420 nm. At the location of the emitter ' $A$ ' and ' $B$ ' height of the flake is $310 \mathrm{~nm}$ and $340 \mathrm{~nm}$ respectively.

To locate the quantum emitters precisely, we performed confocal photoluminescence (PL) mapping in mode by which the sample was scanned point by point. Figure 2(d) shows a PL map over an area of $20 \times 20 \mu \mathrm{m}^{2}$ on the $h \mathrm{BN}$ flake. The location of the quantum emitters ' $\mathrm{A}$ ' and ' $\mathrm{B}$ ' are highlighted by dashed circles. The single photon emission nature of these quantum emitters is evident from their second-order autocorrelation measurements indicating $\mathrm{g}^{2}(0)<0.5$ (see Figs. 3 (a) and 3(b)). Figures 2(e) and 2(f) show the PL spectra of these quantum emitters obtained for insulating and metallic $\mathrm{VO}_{2}$. The emission spectra of each quantum emitter consist of a pronounced ZPL accompanied by a weaker phonon assisted emission $(10,17,43)$. An increase in PL intensity as obtained for metallic $\mathrm{VO}_{2}$ compared to insulating $\mathrm{VO}_{2}$ is noticeable for all quantum emitters, which in general suggests a higher photon emission rate i.e., a decrease in emission lifetime, a change in illumination pattern, or any of these. However, in our experiments, a decrease in the emitter lifetime obtained from Hanbury Brown and Twiss (HBT) measurements for metallic $\mathrm{VO}_{2}$ compared to insulating $\mathrm{VO}_{2}$ suggests that this increase in absolute PL intensity originates from enhancement of emission rate. Recent experiment has reported that the decay rate of $h \mathrm{BN}$ quantum emitters remains constant even when heated up to $800 \mathrm{~K}$ (44) which further corroborates that the enhancement of decay rate is due to modification in LDOS rather than a thermal effect. The defectbased quantum emitter dimensions are at the atomic scale, and thus the lateral position can be precisely measured down to few tens of nm when a sufficient number of photons is collected by the camera along with low noise (readout and amplification) and background signals.

To investigate the single-photon emission characteristics and decay lifetime of the quantum emitters, we measured their second-order intensity correlation functions $g^{2}(\tau)$ in both insulating and metallic phases of $\mathrm{VO}_{2}$. In order to reduce the influence of background signal and noise, we corrected the raw $g_{\text {raw }}^{2}(\tau)$ using the function $g^{2}(\tau)=\left[g_{\text {raw }}^{2}(\tau)-\left(1-\rho^{2}\right)\right] / \rho^{2}$, where $\rho=$ 
$S /(S+B)$ where $S$ and $B$ refer to the signal and the background counts, respectively. This background corrected $g^{2}(\tau)$ was fitted with double exponential of the form (20)

$$
g^{2}(\tau)=1-\rho^{2}\left[(1+\zeta) e^{-\gamma_{1}|\tau|}-\zeta e^{-\gamma_{2}|\tau|}\right]
$$

where, $\zeta, \rho, \gamma_{1,2}$ are laser power-dependent parameters $(20,48)$. Here, $\gamma_{1}$ and $\gamma_{2}$ are the faster and the slower decay time constants, respectively, for a three-level system. The second-order intensity correlation functions $g^{2}(\tau)$ under continuous wave excitation pumping for the quantum emitters ' $A$ ' and ' $B$ ' is shown in Figs. 3(a) and 3(b) respectively, when $\mathrm{VO}_{2}$ is insulating (blue dots) and metallic phase (red dots). The data for the metallic phase $\mathrm{VO}_{2}$ configuration has been offset vertically for visual clarity. Equal-time coincidence counts $g^{2}(0)$ for each quantum emitter is less than 0.5 , which indicates the presence of a single emitter. All measurements were performed at a constant $50 \mu \mathrm{W}$ pump laser power which is orders of magnitude smáller compared to the saturation power of $\sim \mathrm{mW}$ for $h \mathrm{BN}$ quantum emitters $(10,18,19)$. Given the Fresnel reflections from all interfaces, which were analyzed using full-wave simulations, the excitation power within the flake along the axial direction is position dependent. From fitting our experimental data of correlation functions $g^{2}(\tau)$, we extracted the decay constants $\left(\gamma_{1,2}\right)$ which has contributions from the spontaneous decay rates and the pump rate $(45,46)$. The spontaneous decay rates $(\gamma)$ of the emitters ' $A$ ' and 'B' are shown in the Table T1 (see supplementary information section S2 for details). The correlation functions over long-time scales are shown in Fig. S10. With an excitation power of $50 \mu \mathrm{W}$, the pump rates are $\sim 25$-fold and 73-fold slower than the spontaneous decay rates for emitters ' $\mathrm{A}$ ' and ' $\mathrm{B}$ ', respectively, and thus make negligible contributions to the decay constants. From Table T1, we clearly see that for all the emitters, the decay rates are higher in the presence of a metallic- $\mathrm{VO}_{2}$ when compared to an insulating- $\mathrm{VO}_{2}$ configuration. Thus, the emitters ' $\mathrm{A}$ ' and ' $\mathrm{B}$ ' are located at distances, from the surface of $\mathrm{VO}_{2}$, such that their optical environment is modified when $\mathrm{VO}_{2}$ undergoes an insulator-to-metal transition.

To model the distance-dependence of the quantum emitter lifetime on $\mathrm{VO}_{2}$ phase, we define the ratio of their decay rates in the insulating and metallic phases as $\beta$. Figures 3(c) and 3(d) show the two-dimensional plot of relative decay rate $\beta$ as a function of distance $d$ from the $h \mathrm{BN} / \mathrm{VO}_{2}$ interface and the polar orientation angle $\theta$ of the dipole for each quantum emitters ' $\mathrm{A}$ ' and ' $\mathrm{B}$ ' respectively. Each plot has three contour lines; a dashed line for the relative decay rates $\beta$ while the upper and the lower solid contour lines correspond to the error in decay rate $( \pm \Delta \beta)$. 
Using the experimental values of $\gamma$ from the Table T1, we obtained the relative decay rates $\beta$ for the quantum emitters ' $A$ ' and 'B', as $0.818 \pm 0.108$ and $0.800 \pm 0.124$ respectively. From these simulations and the experimental values of $\beta$, it is evident that the quantum emitters are located within a narrow region at a distance $d \sim 21 \mathrm{~nm}$ from the surface of $h \mathrm{BN} / \mathrm{VO}_{2}$ interface. However, the uncertainty in the axial position depends on the emitters' polar angle $\theta$. For emitter ' $A$ ', uncertainty (full width) varies from $\sim 13 \mathrm{~nm}$ at $\theta=0^{0}$ to $\sim 22 \mathrm{~nm}$ at $\theta=90^{\circ}$. Similarly, for quantum emitter ' $\mathrm{B}$ ' uncertainty in their axial location varies from $\sim 15 \mathrm{~nm}$ to $\sim 23 \mathrm{~nm}$ at $\theta=0^{0}$ and $\theta=90^{\circ}$ respectively.

Next, we focus on emission polarimetry of the quantum emitters. Previous studies (47) have shown that the three-dimensional orientation $(\theta, \varphi)$ of a dipole can be directly extracted by analyzing polarization characteristics of its emitted light. Figures $4 \mathrm{a}, 4 \mathrm{~b}$ is the emission polarization measurement from the quantum emitters ' $A$ ' and ' $B$ ' respectively. The data is fitted by the function (47)

$$
I(\alpha)=I_{\min }+\left(I_{\max }-I_{\min }\right) \cos ^{2}(\alpha-\varphi)
$$

where $I_{\min , \max }$ and $\varphi$ are the fitting parameters. From the fit, we obtained for emitter ' $\mathrm{A}$ ': $I_{\min }=$ $0.356 \pm 0.013, I_{\max }=0.966 \pm 0.024$ and $\varphi=175.7^{0} \pm 1.0^{0}$. Similarly, for emitter 'B': $I_{\min }=0.318 \pm 0.034, I_{\max }=0.888 \pm 0.066$ and $\varphi=109.9^{0} \pm 2.9^{0} . \quad$ In emission polarimetry, the polar angle $\theta$ can be extracted from the degree of polarization of the emission defined as

$$
\delta(\theta)=\frac{I_{\max }-I_{\min }}{I_{\max }+I_{\min }}
$$

From the fitting parameters $\left(I_{\max }, I_{\min }\right)$, we obtained $\delta=0.461 \pm 0.023$ and $\delta=0.473 \pm$ 0.070 for emitter ' $A$ ' and 'B' respectively. Figures $4(c)$ and 4(d) show the calculated degree of polarization $\delta$ as a function of the polar orientation angle $\theta$ using the experimental values of numerical aperture (0.9), the refractive indices of $h \mathrm{BN}, \mathrm{VO}_{2}$ (insulating phase) and sapphire. The distance $d$ of the quantum emitters ' $\mathrm{A}$ ' and 'B' from $\mathrm{VO}_{2} /$ Sapphire substrate was set to $d \sim 20 \mathrm{~nm}$. From Figures 4(e) and 4(f) we clearly see that variation in the distance $d$ is negligible (dashed line). The red dot in Figures 4(e) and 4(f) represents the measured value of $\delta$ and we extract the polar orientation angle $\theta=20.5^{\circ} \pm 3.6^{\circ}$ and $\theta=21.2^{0} \pm 4.5^{0}$ for emitter ' $A$ ' and ' $\mathrm{B}$ ' 
respectively. In estimating the value of error in $\theta$ we accounted for the error in location $d$, which is shown Figures 4(e) and 4(f) by solid lines. Figure 4(e) shows nanometer-scale axial localization of emitter 'A' with an uncertainty (full-width) of $\sim 15 \mathrm{~nm}$, oriented along $(\theta, \varphi)=\left(20.5^{0} \pm\right.$ $3.6^{0}, 175.7^{0} \pm 1.0^{0}$ ). Similarly, Figure 4(f) shows nanometer-scale axial localization of emitter ' $\mathrm{B}$ ' with an uncertainty full-width of $\sim 17 \mathrm{~nm}$, oriented along $(\theta, \varphi)=\left(21.2^{0} \pm 4.5^{0}, 109.9^{0} \pm\right.$ $\left.2.9^{0}\right)$. The strong vertical component of the dipole orientation for both emitters found in the vicinity of $h \mathrm{BN} / \mathrm{VO}_{2}$ interface are consistent with our simulations results shown in Figure S6. The predominant out of plane dipole orientation of these emitters can be a result of an out of plane atomic structure of the emitter as discussed in the literature $(48,49)$. We believe the information provided by these measurements are of fundamental value in determining the underlying atomic structure of emitters.

Next, we investigated the feasibility of transferring the $h \mathrm{BN}$ flake from the $\mathrm{VO}_{2}$ measurement substrate to a device substrate, which is a necessary capability for integration of quantum emitters with chip-based photonic components, waveguide circuits, and planar metamaterials (50-53). In contrast to wet chemical transfer method (54), we utilized a polymerassisted hot pickup technique $(30,55,56)$ to transfer the emitter-host $h \mathrm{BN}$ flake from $\mathrm{VO}_{2}$ to a receiving $\mathrm{SiO}_{2} / \mathrm{Si}$ device substrate (Supplementary Figure S13). Figure 5(a), 5(b) show the optical image of the flake before and after the transfer. We were able to relocate the emitters ' $\mathrm{A}$ ' and ' $\mathrm{B}$ ' on the device substrate by performing confocal PL mapping (Fig. 5(c)) and matching the spectral (Fig. 5(d)) and spatial signatures of both emitters before and after the transfer process. We expect that the axial position of the emitters from the bottom surface and the polar angle of their dipole orientation to remain unchanged after the transfer. However, the azimuthal angle may change. Next, we performed emission polarimetry to determine the azimuthal angle of the emitters after the transfer. Figures 5(d) and 5(e) show polar plots of the PL intensity of the emitter 'A' and emitter ' $\mathrm{B}$ ' respectively as a function of the emission polarization analysis angle $(\alpha)$. From the fit using Eq. (2), we deduce that for emitter A, $\varphi=164.6^{\circ} \pm 3.0^{\circ}$ and for emitter $\mathrm{B}, \varphi=94.4^{\circ} \pm$ $4.0^{\circ}$. Next, we measured the relative orientation of the flake before and after the transfer. The transferred flake is oriented at an angle $\Delta \varphi=-12.6^{\circ}$ with respect to its orientation on $\mathrm{VO}_{2} /$ Sapphire which indicates that the azimuthal orientation of the emitters is also preserved within experimental errors. 


\section{Conclusions}

To summarize, we have demonstrated an experimental technique by which the axial position of quantum emitters in a multi-layered $h \mathrm{BN}$ flake can be extracted with nanometer-scale accuracy by exploiting the modification of photonic density of states using a phase change optical material, $\mathrm{VO}_{2}$. Here, we tailor the optical environment of an emitter in the vicinity of $\mathrm{VO}_{2} / \mathrm{Sapphire}$ substrate which generates a sharply distance-dependent PL response. By performing time-resolved fluorescence spectroscopy, supplemented with emission polarimetry, several specific quantum emitters were identified at an axial distance of $\sim 21 \mathrm{~nm}$ from the $h \mathrm{BN} / \mathrm{VO}_{2}$ interface while also determining their full dipolar orientation $(\theta, \varphi)$. Although, in this work, we focused on locating $h \mathrm{BN}$ quantum emitters with ZPL in the wavelength range $\sim 550-640 \mathrm{~nm}$, presence of other emitters with ZPL outside this range as well as embedded further deeper in the flakes cannot be ruled out. Next, we utilized a polymer-assisted hot pickup technique to transfer the identified $h \mathrm{BN}$

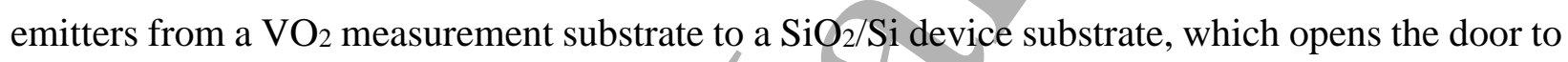
coupling of fully characterized emitters, where each emitter has undergone precise measurement of axial position and orientation. It is worth noting that any phase-change material which experiences a sharp change in optical properties would be suitable for this purpose. However, $\mathrm{VO}_{2}$ is particularly interesting because its insulator-to-metal transition happens near room temperature and is thus well suited to dynamically control emission rates of quantum emitters $(57,58)$ near room temperature. Owing to the broadband nature of change in the dielectric function of $\mathrm{VO}_{2}$ when switched from the insulating to metallic phase, our technique could also be extended to locating other visible or infrared quantum emitters (10). Moving forward, characterizing and controlling the spectral diffusion of our localized $h \mathrm{BN}$ quantum emitters for generating identical photons would constitute an interesting direction for future research.

\section{Experimental Method}

Optical characterization of samples was performed in a home-built confocal microscope capable of optical spectroscopy in visible range (Princeton HRS 300 system) and intensity auto-correlation measurement $\left(\mathrm{g}_{2}(\tau)\right)$ in a Hanbury Brown Twiss (HBT) configuration using a 50-50 beam splitter and two ayalanche photo diodes (PDM Series -PicoQuant). We used a fast-scanning mirror (Newport) and a $4 f$ telecentric configuration to perform photoluminescence mapping. The microscope uses a $532 \mathrm{~nm} \mathrm{CW}$ laser (Cobolt) to pump emitters in $h \mathrm{BN}$, a 100X objective (Leica) 
to focus the beam on the sample and used $50 \mu \mathrm{W}$ power of laser (before objective) for all emitters. A quarter wave plate was put in the beam path at $45^{\circ}$ orientations with respect to linear polarization of laser in order to produce circularly polarized light. We pumped with circularly polarized light to excite all emitters irrespective of their in-plane dipole orientation. A tunable bandpass filter (Semrock versachrome) was used to only pass the zero-phonon line on the emitter into HBT setup to reduce background. Schematic of our optical characterization setup can be seen in Fig. S7. To measure the azimuthal component of the emitter's dipole orientation we put a rotating polarizer in the detection path via a flip mirror and we rotate the polarizer from a vertical configuration with increments of 20 degrees until 340 degrees and the intensity after the polarizer is recorded with an APD. A polar plot of intensity as a function of angle is plotted (Figs. 4(a), 4(b), 5(e), and 5(f)). Visibility of the polar plots were used to determine the polar component of the dipole orientation (Figs. 4(c) and 4(d)). Annealing in an inert environment is routinely used to create or activate quantum emitters in diamond as well as $h \mathrm{BN}$. For the $h \mathrm{BN}$ samples described here, we annealed a bulk crystal of $h \mathrm{BN}$ at $950^{\circ} \mathrm{C}$ in a 1 bar pressure argon gas for 30 minutes before exfoliation. We mounted our sample consisting of $h \mathrm{BN} / \mathrm{VO}_{2} / \mathrm{Sapphire}$ on a Peltier heating stage.

\section{Acknowledgments}

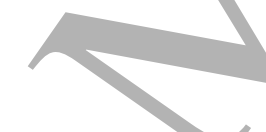

We thank S. Nam for the useful discussions. This work was supported by the DOE "Photonics at Thermodynamic Limits" Energy Frontier Research Center under grant DE-SC0019140 and by the Boeing Company.

\section{Author contributions:}

P. K. J, H. A, Y. K, and H. A. A conceived and developed the idea. P. K. J prepared the $h \mathrm{BN}$ flakes and performed AFM measurements. H. A performed the optical characterization of $h \mathrm{BN}$ flakes and correlation measurements. P. K. J and H. A performed emission polarimetry of $h \mathrm{BN}$ quantum emitters. Y. K prepared $\mathrm{VO}_{2} /$ Sapphire sample; performed ellipsometry, full-wave simulations, optical, and AFM characterizations of $\mathrm{VO}_{2}$ thin films. S. B developed and performed transfer of $h \mathrm{BN}$ flake from $\mathrm{VO}_{2} /$ Sapphire substrate onto $\mathrm{SiO}_{2} / \mathrm{Si}$ for further studies. P. K. J simulated the optical response of $h \mathrm{BN}$ quantum emitters and theoretical estimations with inputs from all co-authors. H.A.A. supervised all the experiments, calculations, and data collection. All authors contributed to the data interpretation, presentation, and writing of the manuscript. 


\section{References and notes}

1. A. D. Franklin, in Point Defects in Solids, J. H. Crawford, L. M. Slifkin, Eds. (Plenum Press, New York, 1972), vol. 1, chap. 1.

2. M. A. Nielsen, I. L. Chuang, Quantum Computation and Quantum Information (Cambridge University Press, 2010).

3. J. R. Weber, W. F. Koehl, J. B. Varley, A. Janotti, B. B. Buckley, C. G. Van de Walle, D. D. Awschalom, Quantum Computing with Defects. Proc. Natl. Acad. Sci. U. S. A. 107, 8513-8518 (2010).

4. A. Beveratos, R. Brouri, T. Gacoin, A. Villing, J.-P. Poizat, P. Grangier, Single Photon Quantum Cryptography. Phys. Rev. Lett. 89, 187901 (2002).

5. C. L. Degen, F. Reinhard, P. Cappellaro, Quantum Sensing. Rev. Mod. Phys. 89, 035002 (2017).

6. M. W. Doherty, N. B. Manson, P. Delaney, F. Jelezko, J. Wrachtrup, L. C. L. Hollenberg, The nitrogen-vacancy colour centre in diamond. Phys. Rep. 528,1-45 (2013).

7. D. D. Awschalom, R. Hanson, J. Wrachtrup, B. B. Zhou, Quantum technologies with optically interfaced solid-state spins. Nat. Photonics 12,516-527(2018).

8. S. Kako, C. Santori, K. Hoshino, S. Götzinger, Y. Yamamoto, Y. Arakawa, A gallium nitride single-photon source operating at 200 K. Nat. Mater. 5, 887- 892 (2006).

9. A. J. Morfa, B. C. Gibson, M. Karg, T. J. Karle, A. D. Greentree, P. Mulvaney, S. Tomljenovic-Hanic, Single-photon emission and quantum characterization of zinc oxide defects. Nano Lett. 12, 949-954 (2012).

10. I. Aharonovich, D. Englund, M. Toth, Solid-State Single-Photon Emitters. Nat. Photonics 10, 631-641(2016).

11. M. Atatüre, D. Englund, N. Vamivakas, S.-Y. Lee, J. Wrachtrup, Material platforms for spin-based photonic quantum technologies. Nat. Rev. Mater. 3, 38-51 (2018).

12. A. Srivastava, M. Sidler, A. V. Allain, D. S. Lembke, A. Kis, A. Imamoğlu, Optically active quantum dots in monolayer WSe2. Nat. Nanotechnol. 10, 491-496 (2015).

13. Y.-M. He, G. Clark, J. R. Schaibley, Y. He, M.-C. Chen, Y.-J. Wei, X. Ding, Q. Zhang, W. Yao, X. Xu, C.-Y. Lu, Jian-Wei Pan, Single quantum emitters in monolayer semiconductors. Nat. Nanotechnol. 10, 497-502 (2015).

14. M. Koperski, K. Nogajewski, A. Arora, V. Cherkez, P. Mallet, J.-Y. Veuillen, J. Marcus, P. Kossacki1, M. Potemski1, Single photon emitters in exfoliated WSe2 structures. Nat. Nanotechnol. 10, 503-506 (2015).

15. C. Chakraborty, L. Kinnischtzke, K. M. Goodfellow, R. Beams, A. Nick Vamivakas, Voltage-controlled quantum light from an atomically thin semiconductor. Nat. Nanotechnol. 10, 507-511 (2015).

16. P. Tonndorf, R. Schmidt, R. Schneider, J. Kern, M. Buscema, G. A. Steele, A. CastellanosGomez, H. S. J. van der Zant, S. M. De Vasconcellos, R. Bratschitsch, Single-photon emission from localized excitons in an atomically thin semiconductor. Optica 2, 347-352 (2015). 
17. T. T. Tran, K. Bray, M. J. Ford, M. Toth, I. Aharonovich, Quantum Emission from Hexagonal Boron Nitride Monolayers. Nat. Nanotechnol. 11, 37-41 (2016).

18. I. Aharonovich, M. Toth, Quantum Emitters in Two Dimensions. Science 358, 170-171 (2017).

19. J. D. Caldwell, I. Aharonovich, G. Cassabois, J. H. Edgar, B. Gil, D. N. Basov, Photonics with Hexagonal Boron Nitride. Nat. Rev. Mater. 4, 552-567(2019).

20. A. L. Exarhos, D. A. Hopper, R. R. Grote, A. Alkauskas, L. C. Bassett, Optical Signatures of Quantum Emitters in Suspended Hexagonal Boron Nitride. ACS Nano 11, 3328-3336 (2017).

21. G. Noh, D. Choi, J.-H. Kim, D.-G. Im, Y.-H. Kim, H. Seo, J. Lee, Stark Tuning of SinglePhoton Emitters in Hexagonal Boron Nitride. Nano Lett. 18, 4710-4715 (2017).

22. Y. Xia, Q. Li, J. Kim, W. Bao, C. Gong, S. Yang, Y. Wang, X. Zhang, Room-Temperature Giant Stark Effect of Single Photon Emitter in van/der Waals Material. Nano Lett. 19, 7100-7105 (2019).

23. N. Nikolay, N. Mendelson, N. Sadzak, F. Böhm, T., T. Tran, B. Sontheimer, I. Aharonovich, O. Benson, Very Large and Reversible Stark-Shift Tuning of Single Emitters in Layered Hexagonal Boron Nitride. Phys. Rev. Applied 11, 041001 (2019).

24. A. L. Exarhos, D. A. Hopper, R. N. Patel, M. W. Doherty, L. C. Bassett, Magnetic-fielddependent quantum emission in hexagonal boron nitride at room temperature. Nat Commun. 10, 222 (2019).

25. A. Gottscholl, M. Kianinia, V. Soltamov, S. Orlinskii, G. Mamin, C. Bradac, C. Kasper, K. Krambrock, A. Sperlich, M. Toth, I. Aharonovich, V. Dyakonov. Initialization and readout of intrinsic spin defects in a van der Waals crystal at room temperature. Nat. Mater. 19, 540-545 (2020).

26. F. Hayee, L. Yu, J. L. Zhang C. J. Ciccarino, M. Nguyen, A. F. Marshall, I. Aharonovich, J. Vučković, P. Narang, T. F. Heinz, J. A. Dionne, Revealing multiple classes of stable quantum emitters in hexagonal boron nitride with correlated optical and electron microscopy. Nat. Mater. 19, 534-539 (2020).

27. A. Dietrich, M. W. Doherty, I. Aharonovich, A. Kubanek, Solid-state single photon source with Fourier transform limited lines at room temperature. Phys. Rev. B 101, 081401(R) (2020).

28. S. A. Tawfik, A. Ali, M. Fronzi, M. Kianinia, T. T. Tran, C. Stampfl, I. Aharonovich, M. Toth, M J. Ford, First-principles investigation of quantum emission from hBN defects. Nanoscale 9, 13575-13582(2017).

29. H. Akbari, W.-H. Lin, B. Vest, P. K. Jha, and H. A. Atwater, Temperature-dependent spectral emission of hexagonal boron nitride quantum emitters on conductive and dielectric substrates. Phys. Rev. Applied 15, 014036 (2021).

30. K. Kang, K.-H. Lee, Y. Han, H. Gao, S. Xie, D. A. Muller, J. Park, Layer-by-layer assembly of two-dimensional materials into wafer-scale heterostructures. Nature 550, 229233 (2017). 
31. C. M. Went, J. Wong, P. R. Jahelka, M. Kelzenberg, S. Biswas, M. S. Hunt, A. Carbone, H. A. Atwater, A new metal transfer process for van der Waals contacts to vertical Schottky-junction transition metal dichalcogenide photovoltaics. Sci. Adv, 5, eaax6061 (2019).

32. J-M Cai, A. Retzker, F. Jelezko, M.-B. Plenio, A large-scale quantum simulator on a diamond surface at room temperature. Nat Phys. 9, 168 (2013).

33. J. L. O’Brien, A. Furusawa, J. Vučković, Photonic Quantum Technologies. Nat. Photonics 3, 687-695 (2009).

34. S. Wehner, D. Elkouss, R. Hanson, Quantum internet: A vision for the road ahead. Science 362, eaam9288 (2018).

35. W. L. Barnes, S. A. R. Horsley, W. L. Vos, Classical antennae, quantum emitters, and densities of optical states. J. Opt. 22, 073501 (2020).

36. K. H. Drexhage in Progress in Optics, E. Wolf Ed. (Êlsevier, New York, 1974), vol XII, pages 163-232.

37. P. K. Jha, M. Mrejen, J. Kim, C. Wu, Y. Wang, Y. V. Rostovtsev, Xiang Zhang, Coherence-Driven Topological Transition in Quantum Metamaterials. Phys. Phys. Lett. 116, 165502 (2016).

38. M. Nalabothula, P. K. Jha, T. Low, A. Kumar, Engineering valley quantum interference in anisotropic van der Waals heterostructures. Phys. Rev. B 102, 045416(2020).

39. A. Ghosh, A. Sharma, A. I. Chizhik, S. Isbaner, D. Ruhlandt, R. Tsukanov, I. Gregor, N. Karedla, J. Enderlein, Graphene-based metal-induced energy transfer for sub-nanometer optical localization. Nat. Photonics 13, 860-865 (2019).

40. M. M. Qazilbash, M. Brehm, B. G. Chae, P. C. Ho, G. O. Andreev, B. J. Kim, S. J. Yun, A. V. Balatsky, M. B. Maple, F. Keilmann, H. T. Kim, D. N. Basov, Mott transition in $\mathrm{VO}_{2}$ revealed by infrared spectroscopy and nano-imaging. Science 318, 1750-1753 (2007).

41. D. Golla, K. Chattrakun, K. Watanabe, T. Taniguchi, B. J. LeRoy, A. Sandhu, Optical thickness determination of hexagonal boron nitride flakes. Appl. Phys. Lett. 102, 161906 (2013).

42. N. Nikolay, N. Mendelson, E. Özelci, B. Sontheimer, F. Böhm, G. Kewes, M. Toth, I. Aharonovich, O. Benson, Direct measurement of quantum efficiency of single-photon emitters in hexagonal boron nitride. Optica 6, 1084-1088 (2019).

43. D. Wigger, R. Schmidt, O. D. Pozo-Zamudio, J. A. Preuß, P. Tonndorf, R. Schneider, P. Steeger, J. Kern, Y. Khodaei, J. Sperling, S. M. de Vasconcellos, R. Bratschitsch, and T. Kuhn. Phonon-assisted emission and absorption of individual color centers in hexagonal boron nitride. 2D Material 6, 035006 (2019).

44. M. Kianinia, B. Regan, S. A. Tawfik, T. T. Tran, M. J. Ford, I. Aharonovich, M. Toth, Robust Solid-State Quantum System Operating at 800 K. ACS Photonics 4, 768-773 (2017). 
45. E. Wu, V. Jacques, H. Zeng, P. Grangier, F. Treussart, J.-F. Roch, Narrow-band singlephoton emission in the near infrared for quantum key distribution. Opt. Express 14, 12961303 (2006).

46. C. C. Davis, Laser and Electro-optics: Fundamental and Engineering (Cambridge University Press, 2014).

47. C. Lethiec, J. Laverdant, H. Vallon, C. Javaux, B. Dubertret, J.-M. Frigerio, C. Schwob, L. Coolen, A. Maître, Measurement of Three-Dimensional Dipole Orientation of a Single Fluorescent Nanoemitter by Emission Polarization Analysis. Phys. Rev, X 4, 021037 (2014).

48. N. Mendelson, D. Chugh, J. R. Reimers, T. S. Cheng, A. Gottscholl, H. Long, C. J. Mellor, A. Zettl, V. Dyakonov, P. H. Beton, S. V. Novikov, C. Jagadish, H. H. Tan, M. F. Ford, M. Toth, C. Bradac, I. Aharonovich, Identifying carbon as the source of visible singlephoton emission from hexagonal boron nitride. Nat. Mater. 20, 321-328(2021).

49. C. Lyu, Y. Zhu, P. Gu, J. Qiao, K. Watanabe, T. Taniguchi, Y. Ye, Single-photon emission from two-dimensional hexagonal boron nitride annealed in a carbon-rich environment. Appl. Phys. Lett. 117, 244002 (2020).

50. P. K. Jha, X. Ni, C. Wu, Y. Wang, X. Zhang, Metasurface-Enabled Remote Quantum Interference. Phys. Rev. Lett. 115, 025501 (2015).

51. P. K. Jha, N. Shitrit, J. Kim, X. Ren, Y. Wang, X. Zhang, Metasurface-Mediated Quantum Entanglement. ACS Photonics 5, 971-976 (2017).

52. P. K. Jha, N. Shitrit, X. Ren, Y. Wang, Xiang Zhang, Spontaneous Exciton Valley Coherence in Transition Metal Dichalcogenide Monolayers Interfaced with an Anisotropic Metasurface. Phys. Rev. Lett. 121, 116102 (2018).

53. X. Ren, P. K. Jha, Y. Wang, X. Zhang. Non-Conventional Metasurfaces: from nonHermitian coupling, quantum interactions, to skin cloak. Nanophotonics 7, 1233 (2018).

54. T. Vogl, G. Campbell, B. C. Buchler, Y. Lu, P. K. Lam, Fabrication and Deterministic Transfer of High-Quality Quantum Emitters in Hexagonal Boron Nitride. ACS Photonics 5, 2305-2312 (2018).

55. F. Pizzocchero, L. Gammelgaard, B. Jessen, J. M. Caridad, L. Wang, J. Hone, P. Bøggild, T. J. Booth, The hot pick-up technique for batch assembly of van der Waals heterostructures. Nat Commun 7, 11894 (2016).

56. D. G. Purdie, N. M. Pugno, T. Taniguchi, K. Watanabe, A. C. Ferrari, A. Lombardo, Cleaning interfaces in layered materials heterostructures. Nat Commun 9, 5387 (2018).

57. S. Cueff, D. Li, Y.Zhou, F. J. Wong, J. A. Kurvits, S. Ramanathan, R. Zia, Dynamic control of light emission faster than the lifetime limit using $\mathrm{VO}_{2}$ phase-change. Nat. Commun. 6, 8636 (2015).

58. D. Szilard, W. J. M. Kort-Kamp, F. S. S. Rosa, F. A. Pinheiro, C. Farina, Hysteresis in the spontaneous emission induced by $\mathrm{VO}_{2}$ phase change. J. Opt. Soc. Am. B 36, C46-C51 (2019). 
59. O. Stenzel, J. Hahn, M. Roder, A. Ehrlich, S. Prause, and F. Richter, The optical constants of cubic and hexagonal boron nitride thin films and their relation to the bulk optical constants. Phys. Status Solidi A 158, 281-287 (1996).

60. E. D. Palik, Handbook of Optical Constants of Solids: Index (Academic Press, Waltham, MA, 1991).

61. Y. Kim, P. C. Wu, R. Sokhoyan, K. Mauser, R. Glaudell, G. K. Shirmanesh, and H. A. Atwater, Phase Modulation with Electrically Tunable Vanadium Dioxide Phase-Change Metasurfaces. Nano Lett. 19, 2961-3968 (2019).

62. M. K. Boll, I. P. Radko, A. Huck, and U. L. Andersen, Photophysics of quantum emitters in hexagonal boron-nitride nano-flakes. Opt. Exp. 28, 7475 (2020). 
a)

Figure 1: Experimental schematic and distance-dependent modulation of relative decay rates. (a) Schematic of a quantum emitter in an atomically thin crystal of hexagonal boron nitride $(h \mathrm{BN})$ located within the thickness of a flake on a substrate which consists of a thin layer of vanadium dioxide $\left(\mathrm{VO}_{2}\right)$ deposited on a sapphire crystal. (b) Relative decay rate $\beta=\gamma_{\text {Insulating }} / \gamma_{\text {Metallic }}$ as a function of distance $d$ of a quantum emitter from the surface of $\mathrm{VO}_{2}$ when switched from the insulating to metallic state. The blue and red curves refer to quantum emitters oriented parallel and perpendicular to the surface respectively and the shaded regions corresponds to the typical quantum yield (QY) range of 0.6-1.0 of $h \mathrm{BN}$ quantum emitters with zero-phonon line around 600 $\mathrm{nm}$ (42). For numerical simulation we considered the emission wavelength of $600 \mathrm{~nm}$ for the quantum emitter corresponding to the emitter 'A' (Fig. 2e). The dashed line within the shaded region corresponds to $\mathrm{QY}=0.79$ as estimated from our experimental data (Supplementary Sec. $\mathrm{S} 2)$. The refractive indices of the upper medium, $h \mathrm{BN}$, and that of sapphire were set to $1,1.82$ (59), and 1.77 (60) respectively. The refractive index of $\mathrm{VO}_{2}$ at $600 \mathrm{~nm}$ was set to $3.05+0.42 \mathrm{i}$ (insulating state) and $2.57+0.64 \mathrm{i}$ (metallic state) from our ellipsometric data (Supplementary Fig. S1). 

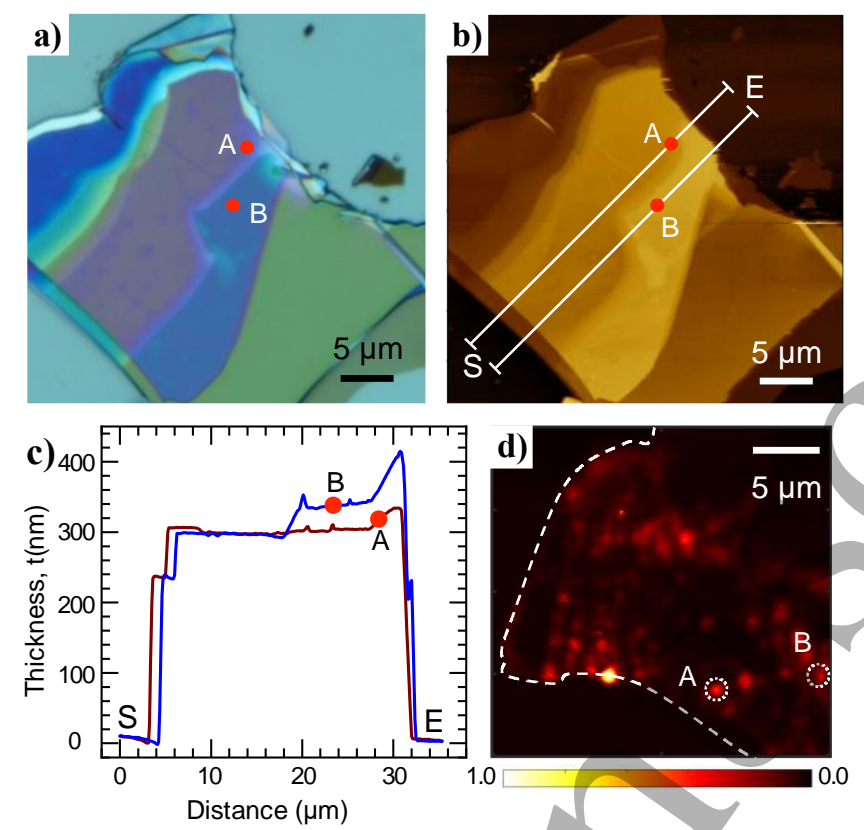

e)

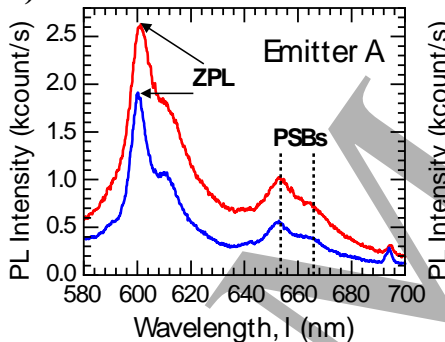

f)

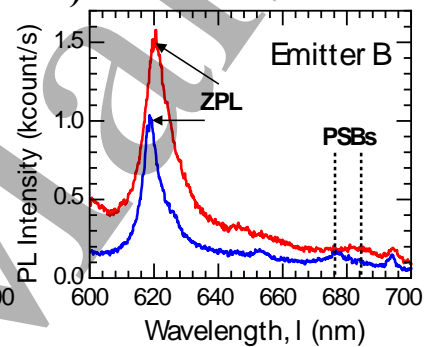

Figure 2: Characterization of the exfoliated flake and spectra of $h \mathrm{BN}$ quantum emitters. (a) Optical image of the mechanically exfoliated $h \mathrm{BN}$ flake on $\mathrm{VO}_{2} /$ Sapphire substrate. (b) Atomic force microscopy image of the flake shown in (a). The red dots on the traces (S-E) in (b) and (a) indicate the position of emitters ' $A$ ', and 'B' with emission wavelength of $600 \mathrm{~nm}$ and $620 \mathrm{~nm}$, respectively. (c) Line profiles along the region indicated by the trace in (b). At the location of the emitter ' $A$ ' and ' $B$ ' height of the flake is $310 \mathrm{~nm}$ and $340 \mathrm{~nm}$ respectively. (d) Confocal photoluminescence (PL) map of the $h \mathrm{BN}$ flake. The position of two single photon emitters is marked by white circles. The edge of the flake in marked by white dashed line. The PL spectra of emitters ' $A$ ' and ' $B$ ' shown in (e) and (f) respectively were obtained with $\mathrm{VO}_{2}$ in insulating state (blue) and metallic state (red). In these spectra ZPL and phonon sidebands (PSBs) are highlighted and the small peak around $692 \mathrm{~nm}$ corresponds to the emission from sapphire. 

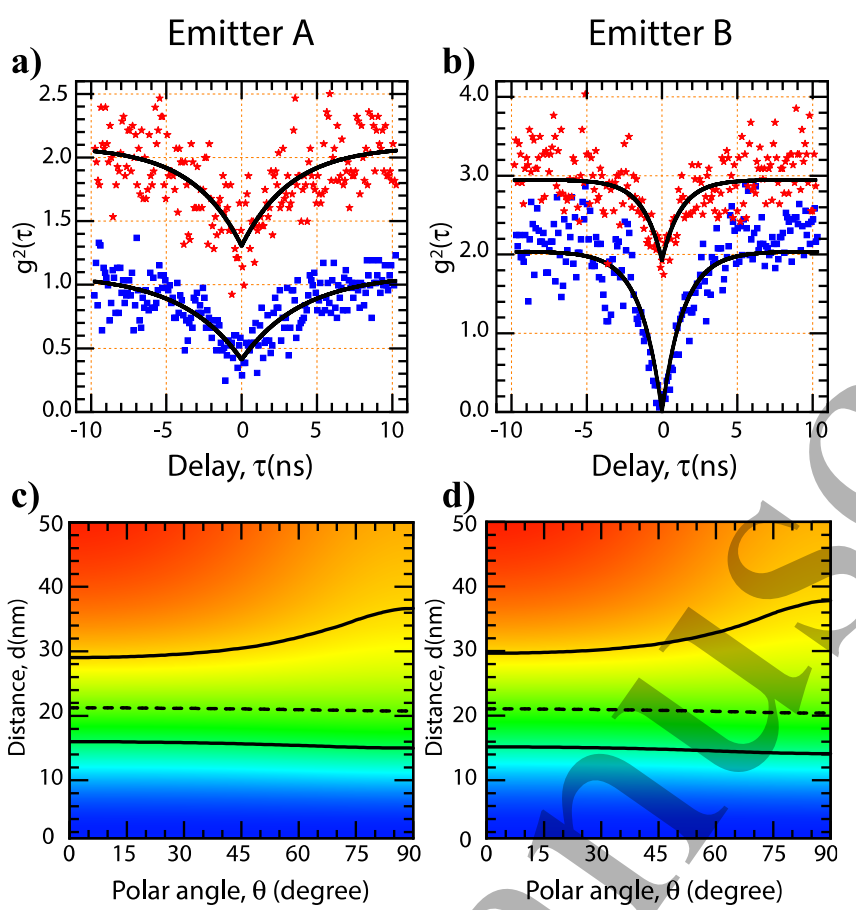

d)

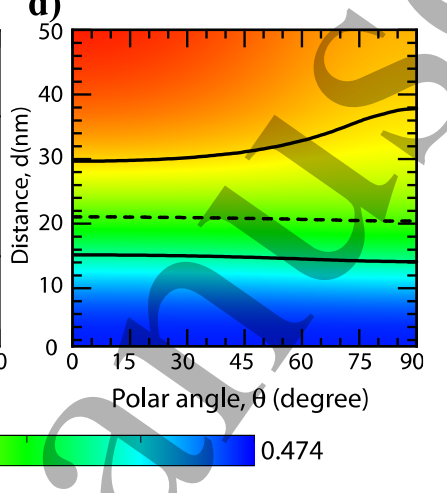

Figure 3: Single photon source characterization and axial location in $h \mathrm{BN}$ flake. Plot of the second order photon correlation measurement, $g^{2}(\tau)$ for the emitters ' $A$ ' and 'B', in (a) and (b) respectively. The experimental data, blue squares for insulating $\mathrm{VO}_{2}$ phase and red stars for metallic $\mathrm{VO}_{2}$, were fitted using Eq. (1) to obtain the decay rates of the emitters. From the fit, we calculated the relative decay rates $\beta=\gamma_{\text {Insulating }} / \gamma_{\text {Metallic }}$ for the three emitters ' $\mathrm{A}$ ' and 'B' as $0.818 \pm 0.108$ and $0.800 \pm 0.124$ respectively. For clarity, $\mathrm{g}^{2}(\tau)$ data obtained for metallic $\mathrm{VO}_{2}$ in (a), and (b) were shifted by 1 and 1.5 respectively. Plot of the relative decay rates $\beta$ as a function of the distance $(d)$ from the surface of $\mathrm{VO}_{2}$ and the polar angle $(\theta)$ for the emitters 'A' and ' $\mathrm{B}$ ' are shown in (c) and (d) respectively. The dashed contour lines in (c) and (d) corresponds to the experimental value of $\beta$ obtained from (a) and (b) respectively, while the solid lines correspond to the error $( \pm \Delta \beta)$ in the ratio. 
Emitter A

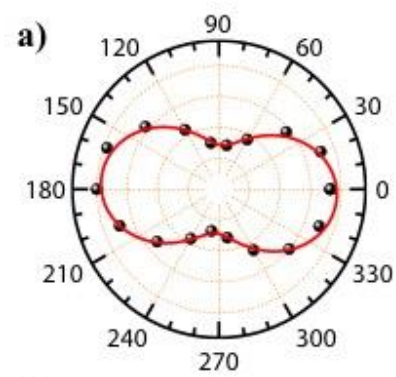

c)
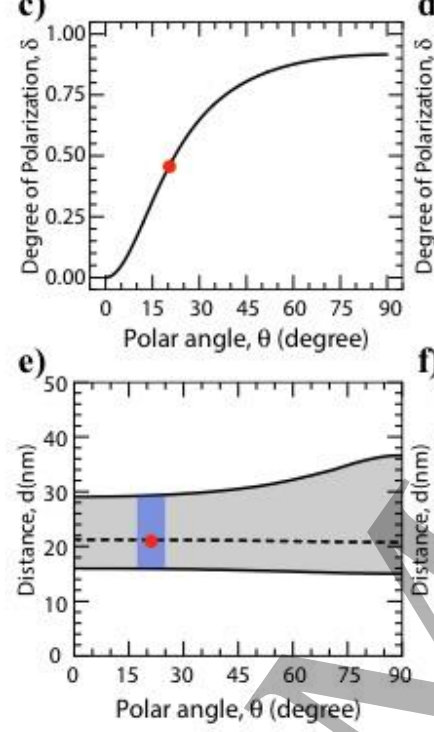

Emitter B

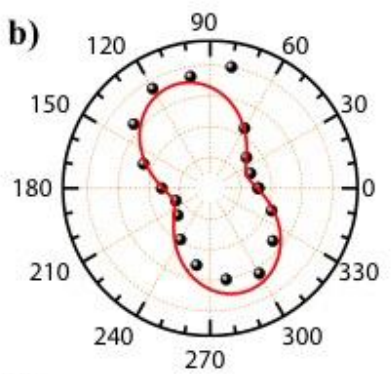

d)
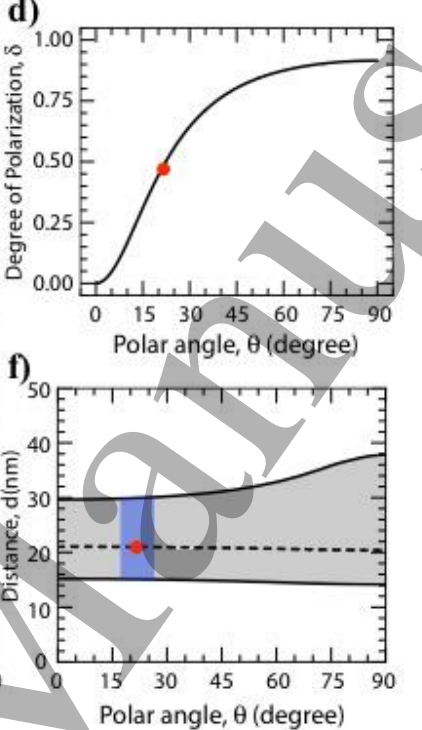

Figure 4: 3D-Orientation of $h \mathrm{BN}$ quantum emitters and nanometric axial location. (a,b) Polar plots of the photoluminescence (PL) intensity of the emitter ' $A$ ' and emitter ' $B$ ' respectively as a function of the emission polarization analysis angle $(\alpha)$. The PL data (solid spheres) were fitted using Eq. (2) to extract the azimuthal angle $(\varphi)$ of the emitters and the degree of polarization $(\delta)$. From the fit, we deduce that for emitter A, $\varphi=175.7^{\circ} \pm 1.0^{\circ} ; \delta=0.461 \pm 0.023$ and for emitter $\mathrm{B}, \varphi=109.9^{\circ} \pm 2.9^{\circ} ; \delta=0.473 \pm 0.070$. (c, d) Calculated value of degree of polarization $(\delta)$ as a function of the polar angle $(\theta)$ of the emitters located at a distance of $\sim 21 \mathrm{~nm}$ from the surface of $\mathrm{VO}_{2}$. The red dots in (c) and (d) corresponds to the experimental value of $\delta$ obtained from (a) and (b) respectively. The extracted value of the polar angle for emitter ' $A$ ' and ' $B$ ' are $\theta=20.5^{\circ}$ $\pm 3.6^{\circ}$ and $\theta=21.2^{\circ} \pm 4.5^{\circ}$ respectively. (e, f) Purple shaded region shows the range of the distance $(d)$ and the polar angle $(\theta)$ of the emitters ' $A$ ' and 'B' respectively based on our experimental data and simulations. 

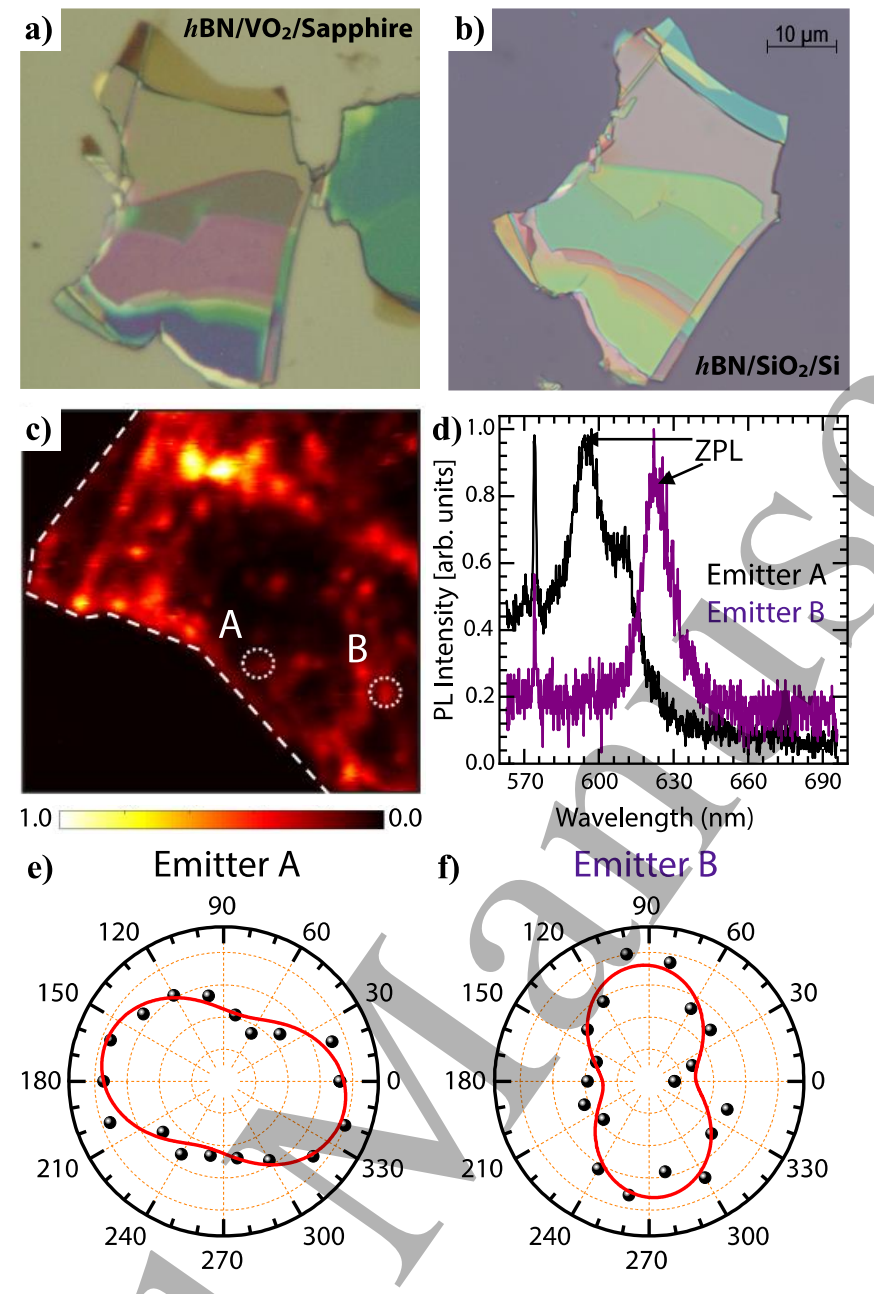

Figure 5: (a,b) Optical image of the flake on $\mathrm{VO}_{2} /$ Sapphire substrate (before transfer) and $\mathrm{SiO}_{2} / \mathrm{Si}$ substrate (after transfer) respectively. The transferred flake is oriented at an angle $\Delta \varphi=-12.6^{\circ}$ with respect to its orientation on $\mathrm{VO}_{2} /$ Sapphire substrate. (c) Confocal photoluminescence (PL) map of the flake on $\mathrm{SiO}_{2} / \mathrm{Si}$ substrate. Encircled (dashed white circle) highlights the region where emitters ' $\mathrm{A}$ ' and ' $\mathrm{B}$ ' are located. (d) PL spectra from the region highlighted in (c) shows the presence of emitters ' $A$ ' and ' $B$ ' which matched with the spectra obtained from the same location before transfer. The sharp line $\sim 575 \mathrm{~nm}$ corresponds to Raman line of $h \mathrm{BN}$. (e,f) Polar plots of the PL intensity of the emitter 'A' and emitter ' $\mathrm{B}$ ' respectively as a function of the emission polarization analysis angle $(\alpha)$ on $\mathrm{SiO}_{2} / \mathrm{Si}$ substrate after the transfer. From the fit using Eq. (2), we deduce that for emitter A, $\varphi=164.6^{\circ} \pm 3.0^{\circ}$ and for emitter $\mathrm{B}$, $\varphi=94.4^{\circ} \pm 4 \cdot 0^{\circ}$. 